- The degree of anterior tooth display is determined by the lips at rest and during smiling.

- The LARS factor guides amount of tooth exposure in the static muscular position of the lips.

- A smile is determined by the dynamic muscular position of the lips.

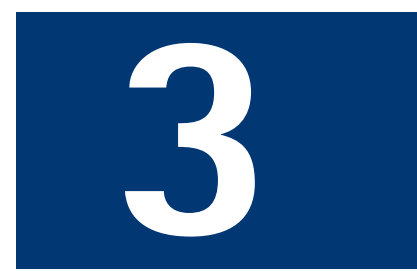

VERIFIABLE CPD PAPER

\title{
Anterior dental aesthetics: Dentofacial perspective
}

I. Ahmad ${ }^{1}$

The purpose of this series is to convey the principles governing our aesthetic senses. Usually meaning visual perception, aesthetics is not merely limited to the ocular apparatus. The concept of aesthetics encompasses both the time-arts such as music, theatre, literature and film, as well as space-arts such as paintings, sculpture and architecture.

\section{ANTERIOR DENTAL AESTHETICS}

1. Historical perspective

2. Facial perspective

3. Dento-facial perspective

4. Dental perspective

5. Gingival perspective

6. Psychological perspective*

* Part 6 available in the $B D J$ book of this series
BDS, The Ridgeway Dental Surgery, 173 The Ridgeway, North Harrow, Middlesex HA2 7DF, United Kingdom.

Tel: +44 (0)20 8861 3535, Fax: +44 (0)20 8861 6181, www.IrfanAhmadTRDS.co.uk Email:iahmadbds@aol.com

\section{Refereed Paper}

doi: 10.1038/sj.bdj.4812412

๑ British Dental Journal 2005; 199 :

81-88

\section{INTRODUCTION}

The third paper in this series on anterior maxillary dental aesthetics discusses the dento-facial view. This perspective concentrates on the orofacial landmarks consisting of highly vascularised lips with the teeth acting as a gateway to the oral cavity. The emphasis here is to analyse

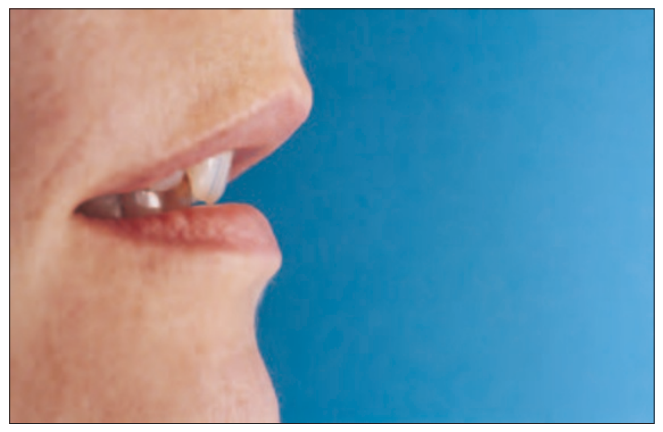

Fig. 1 Sagittal view of the static position of the dento-facial composition with relaxed orofacial muscles

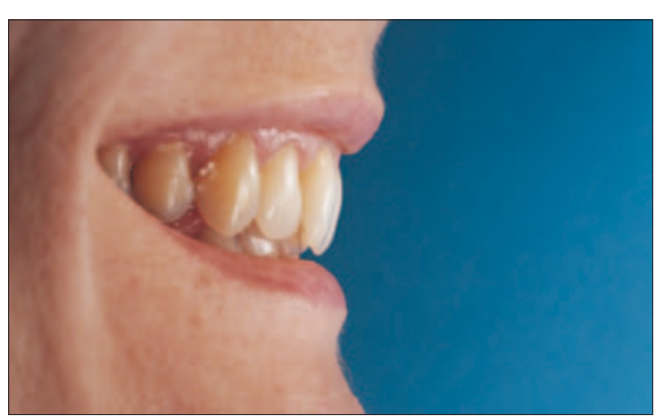

Fig. 2 Sagittal view of the dynamic position of the dento-facial composition with contracted orofacial muscles during a relaxed smile the lips in relation to the anterior maxillary sextant. The deeper colour of the lips compared with lighter coloured teeth creates a colour contrast to add interest to this arrangement. The dento-facial composition encompasses both the frontal and sagittal planes in two muscular positions; the static and dynamic (Figs 1 to 4).

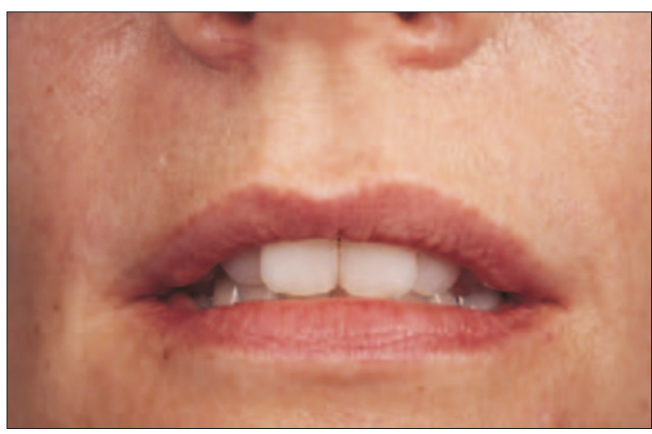

Fig. 3 Frontal view of the static position of the dento-facial composition with relaxed orofacial muscles

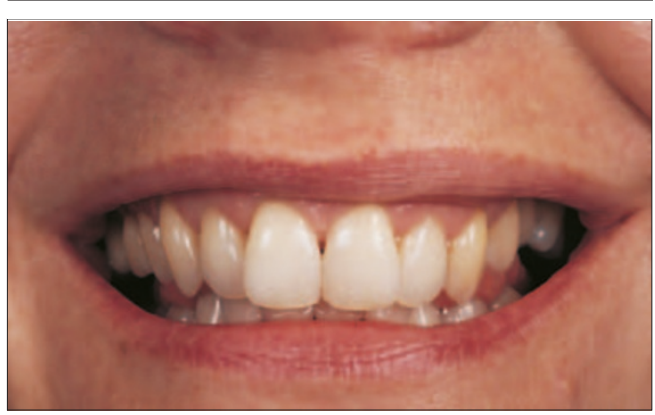

Fig. 4 Frontal view of the dynamic position of the dento-facial composition with contracted orofacial muscles during a relaxed smile 
Fig. 5 The LARS factor to determine the amount of tooth exposure at rest. Lip length: the linear measurement of the upper lip. The patient depicted has a medium maxillary lip length

Fig. 6 The LARS factor to determine the amount of tooth exposure at rest. Age: an elderly individual showing only the lower incisors

Fig. 7 The LARS factor to determine the amount of tooth exposure at rest. Race: black individuals reveal less maxillary teeth than Caucasians

Fig. 8 The LARS factor to determine the amount of tooth exposure at rest. Gender:

females show nearly twice the amount of upper teeth than men

\section{STATIC}

The static position is when the lips are slightly parted and the teeth are out of occlusion with the perioral muscles relatively relaxed. This position is typically achieved following utterance of the letter 'M'. In this tranquil position, four factors influence tooth exposure: lip length, age, race and sex, also known as the acronym LARS (Figs 5-8).,

The length of the upper lip varies from 10$36 \mathrm{~mm}$, and individuals with long maxillary lips show more mandibular rather than maxillary teeth. The amount of tooth exposure at rest is predominantly a muscle-determined position (Table 1).

Table 1 Maxillary lip length in relation to anterior tooth exposure

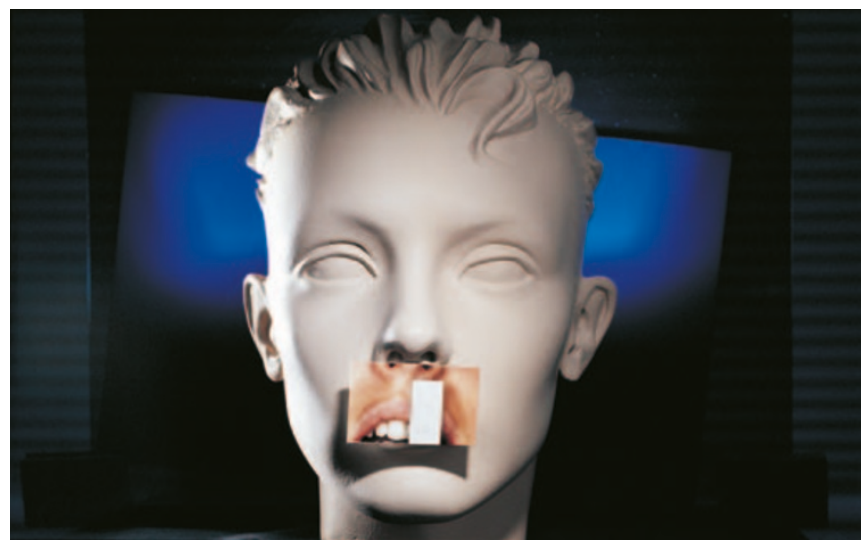

\begin{tabular}{llll}
\hline $\begin{array}{l}\text { Maxillary lip } \\
\text { classification }\end{array}$ & $\begin{array}{l}\text { Maxillary lip } \\
\text { length }(\mathrm{mm})\end{array}$ & $\begin{array}{l}\text { Exposure of } \\
\text { upper } \\
\text { central incisor } \\
(\mathrm{mm})\end{array}$ & $\begin{array}{l}\text { Exposure of } \\
\text { lower } \\
\text { central incisor } \\
(\mathrm{mm})\end{array}$ \\
Short & $10-15$ & 3.92 & 0.64 \\
Medium & $16-20$ & 3.44 & 0.77 \\
Medium & $21-25$ & 2.18 & 0.98 \\
Long & $26-30$ & 0.93 & 1.95 \\
Long & $31-36$ & 0.25 & 2.25 \\
\hline
\end{tabular}

Age is the second part of the LARS factor, which in a similar way to lip length, influences the amount of tooth visibility. The amount of maxillary tooth displayed is inversely proportional to increasing age whereas the amount of mandibular teeth is directly proportional to increasing age. Therefore, a young person will display more maxillary than mandibular teeth, whereas an older individual will show more mandibular, rather than maxillary teeth:

- Maxillary incisor tooth visibility (inversely proportional to age)

- Mandibular incisor tooth visibility (directly proportional to age).

People age at differing rates because ageing is a multi-factorial phenomenon described by the three Ps: programmed, pathological, and pychological ageing.

During youth, the process of destruction and formation of cells is in equilibrium. With advancing years the balance shifts in favour of increased destruction and reduced replacement of body tissues. This change is called programmed or physiological ageing, and is triggered by an internal 'biological clock'. Why and when this change occurs is still unknown.

Pathological ageing is due to diseases of the oral environment leading to accelerated tissue degradation. For example, if anterior teeth are lost due to refractory periodontitis, premature formation of nasolabial grooves is evident.

Finally, when feelings of fatality prevail over those of existentialism ${ }^{3}$, due to emotional and personal traumas, changes in the psyche are observed. These psychosomatic changes manifest themselves as psychological ageing.

The result of the three Ps of ageing is reduced tonicity of the orofacial muscles and laxness of tegumental relief in the lower third of the face resulting in formation of the labial, nasolabial and mental grooves and ridges (Fig. 9). The loss of elasticity of the upper lip, with decreasing tooth support by the gingival $2 / 3$ of the maxillary incisors, accounts for less maxillary and more mandibular incisor tooth display. 


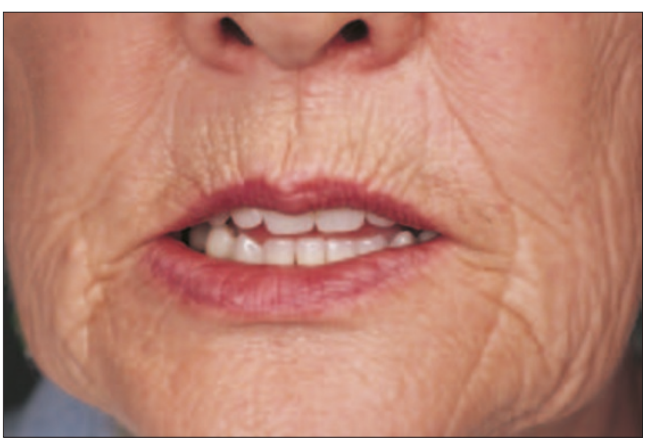

Fig. 9 An edentulous 50-year-old woman showing pathological ageing due to premature loss of all her dentition in her twenties, resulting in development of the nasolabial grooves and ridges

The last two determinants of the LARS factor are race and sex. A decreasing amount of maxillary, and an increasing amount of mandibular tooth visibility, is seen from Caucasians to Asians to Blacks, while the last constituent is the sex of the individual.

Males generally have longer maxillary lips than females, leading to an average maxillary tooth display of $1.91 \mathrm{~mm}$ for men and 3.40 $\mathrm{mm}$ for women. Consequently, gender differences account for females displaying nearly twice the amount of maxillary teeth compared with men.

In conclusion, before the amount of tooth exposure at rest is prescribed for the proposed prostheses, each patient should be assessed according to the LARS factor, e.g. an increased maxillary tooth exposure is indicated for young females, and the opposite for elderly males (Figs 10 and 11).

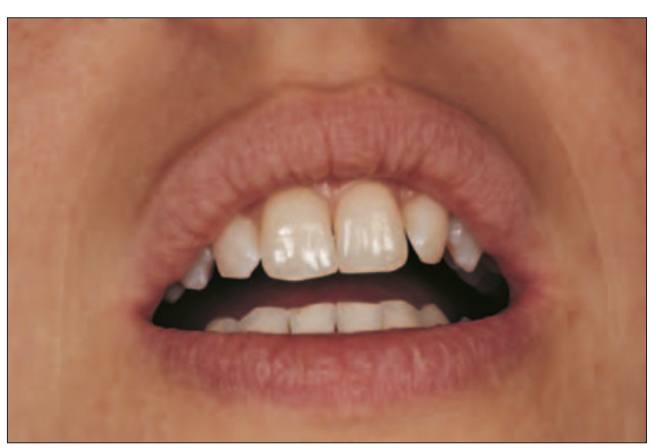

Fig. 10 Young females display a greater degree of the upper anterior teeth due to pronounced tonicity and shorter maxillary lips

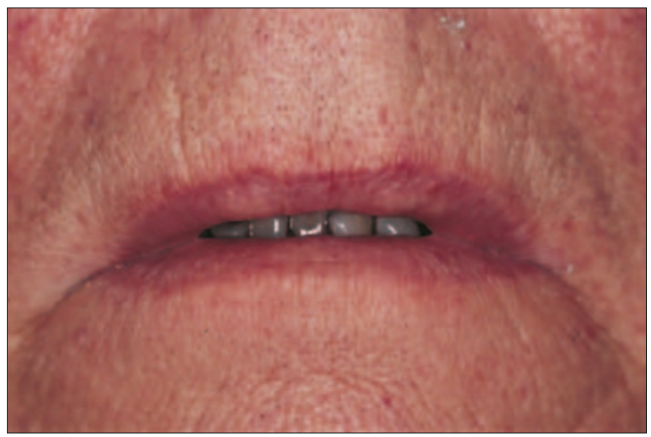

Fig. 11 Elderly males show little, or no, upper teeth but more lower teeth due to tegumental laxness and longer maxillary lips

\section{THE DYNAMIC POSITION}

The second constituent of dento-facial composition is the dynamic position, typically characterised by a smile. The extent of tooth exposure during a smile depends on skeletal make-up, degree of contraction of the facial muscles, shape and size of the dental elements and shape and size of the lips, which vary from extremely thin to full and thick (Figs 12 and 13). According to Rufenacht's morphopsychological concepts, individuals with thin and taut lips should be provided with teeth which confer delicacy and fragility. Conversely, patients endowed with thick or voluptuous lips require teeth which display dominance and boldness.

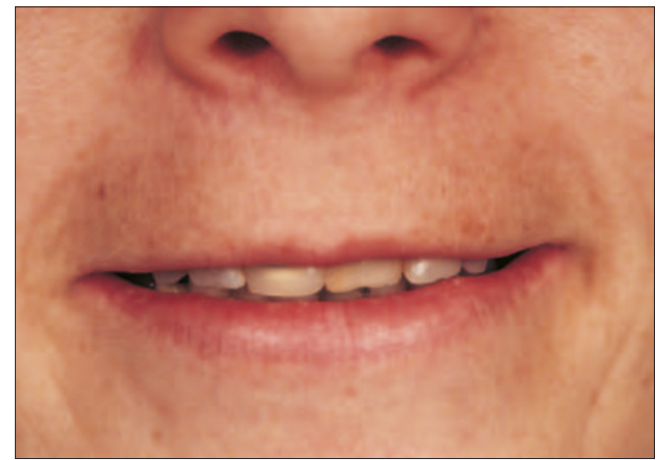

Fig. 12 Frontal view of woman with thin, taut lips

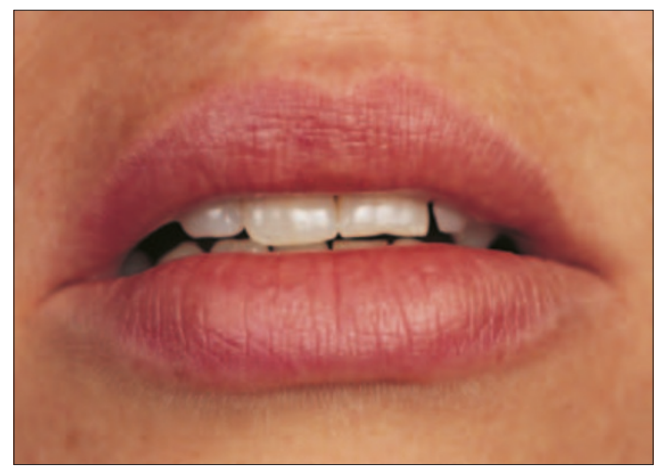

Fig. 13 Frontal view of woman with thick, voluptuous lips

Whereas in the facial composition horizontal symmetry was the most important factor, in the dento-facial view it is radiating symmetry that takes precedence. Radiating symmetry is defined as an object having a central point, from which the right and left sides are mirror images. ${ }^{4}$ In this view, the fulcrum, or central point is the maxillary dental midline, and the right and left upper anterior teeth are balanced mirror images. However, due to differing wear patterns of the incisal edges, this ideal is uncommon. Lack of radiating symmetry is not crucial, so long as there is balance on the right and left sides of the anterior dental segment.

Cohesive forces, as in the facial composition, add interest to the dentofacial composition. These are created by parallelism of the incisal (lime green) and commissural (blue) lines, with the segregative force of the dental midline 
Fig. 14 (top) Coincidence of the incisal plane (lime) with the commissurat line (blue). The yellow line depicts the maxillary dental midline intersecting the horizontal lines at $90^{\circ}$

\section{Fig. 15 (bottom) Lack of} coincidence of the facial midline (red) with the maxillary dental midline (yellow) is not a prerequisite for aesthetic approval (yellow) intersecting at 90 degrees (Fig. 14). Placement of the dental midline has evoked considerable controversy in dental literature. One school of thought states that the maxillary dental midline should coincide exactly with the labial fraenum and the facial midline ${ }^{5}$, as it does in $70 \%$ of the population. The opposing view is that placing the midline exactly in the centre may contribute to a sense of artificiality. ${ }^{6}$ The choice of where to place this midline should be determined after aesthetic appraisal. If a dominant central point of focus exists, e.g. a maxillary median diastema, then the midline should be placed with this focal point as the fulcrum. Another reason for placing a vertically aligned midline precisely in the centre is to detract attention from asymmetries and disharmonies of the face. Opticians and the cosmetic industry, with dramatic visual effect, exploit this concept of guiding the eye to a particular point of focus on the face to mitigate blemishes or undesirable facial features. Alternatively, a slightly off-centre placement of the dental midline in relation to the facial midline is not detrimental to aesthetic approval (Fig. 15). The mandibular midline should not be used as a reference point, because in $75 \%$ of the population this does not coincide with the maxillary midline. ${ }^{7}$
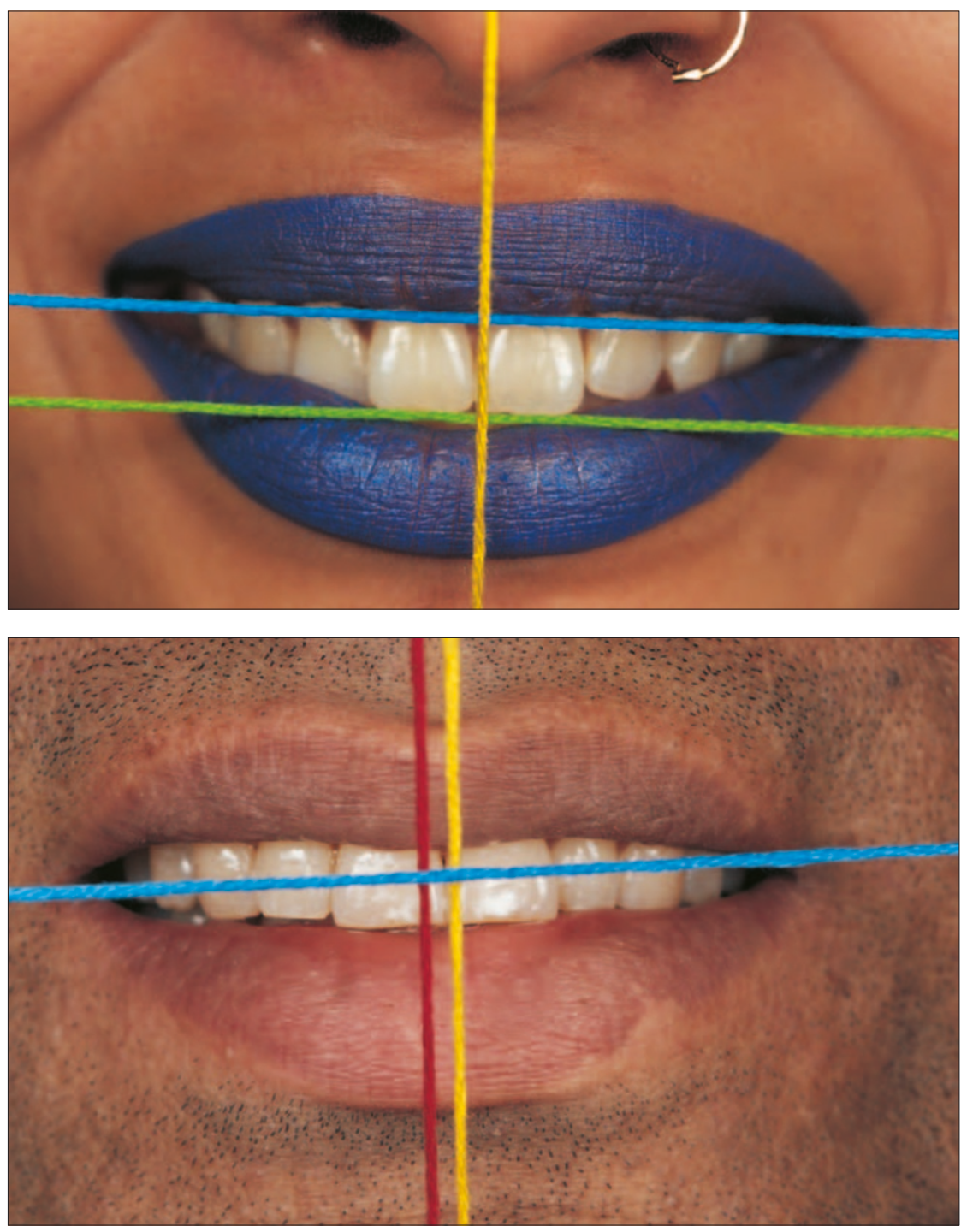

\section{THE SMILE LINE}

The smile line is an imaginary line running from the incisal edges of the maxillary incisors and coinciding with the curvature of the lower lip. When the incisal plane is not parallel with the curvature of the lower lip then flat, eccentric or reversed smile lines are evident. This coincidence between the incisal table and the mandibular lip is often lost due to wear by abrasion, erosion or attrition (Fig. 16), periodontitis (Figs 17 and 18), altered patterns of eruption (Fig. 19) or poor quality dentistry (Figs 20 and 21).

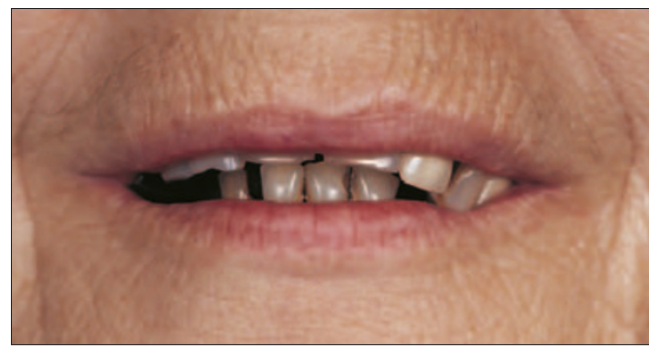

Fig. 16 Lack of parallelism of the maxillary incisal plane with curvature of the mandibular lip due to pronounced wear of the maxillary teeth

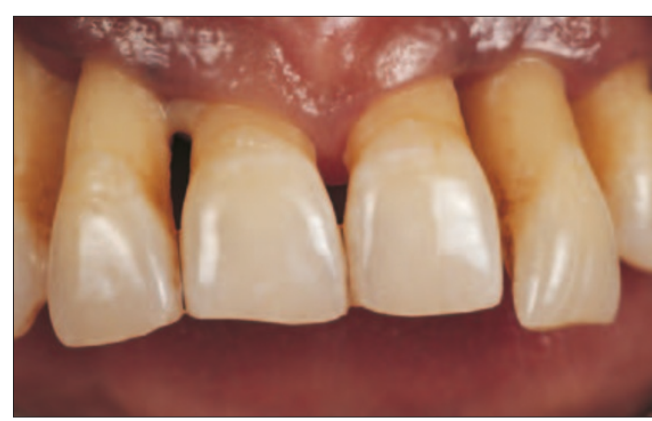

Fig. 17 Refractory periodontitis leading to over-eruption of the lateral incisors leading to disruption of the incisal plane in relation to the mandibular lip curvature (see Fig. 18)

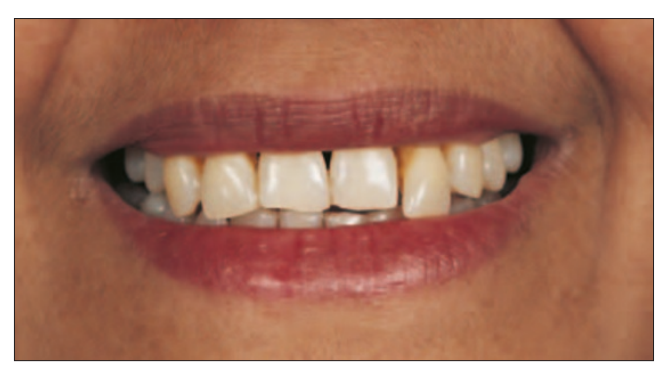

Fig. 18 Dento-facial view of patient in Fig. 17 showing disharmony of the maxillary incisal plane in relation to the mandibular lip curvature

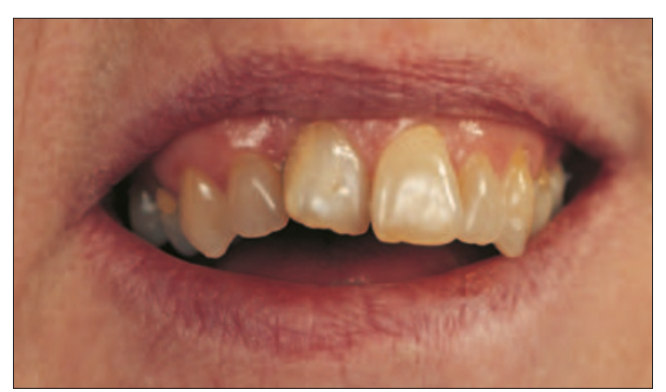

Fig. 19 Altered eruption patterns of maxillary teeth resulting in an eccentric incisal plane, not parallel with the mandibular lip curvature 


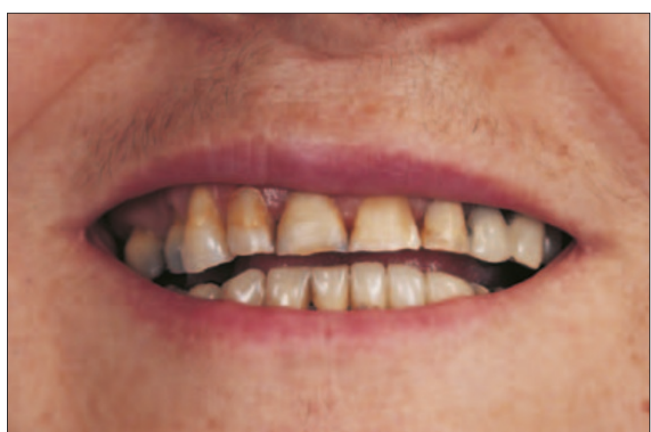

Fig. 20 latrogenic dentistry leading to a revered incisal plane in relation to the mandibular lip curvature

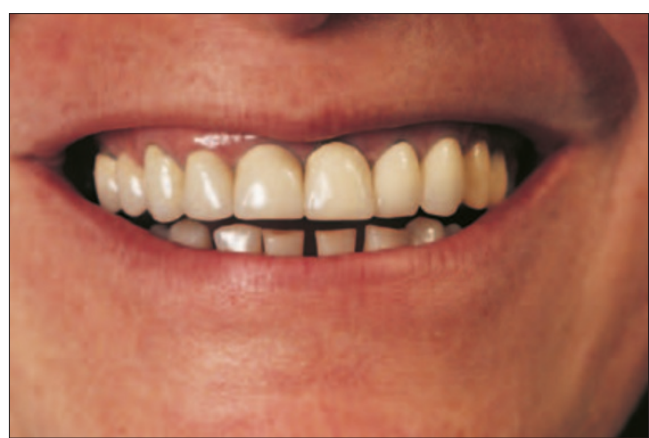

Fig. 21 Flat incisal plane due to short crowns on the central incisors and lack of anterior-posterior incisal embrasure progression

Frequently, this is observed in old removable full dentures, where the acrylic teeth show an uneven wear pattern. This leads to an eccentric smile line, not coincident with the curvature of the mandibular lip. Therefore, replacement of the prostheses with new teeth should endeavour to restore the smile line, ensuring parallelism with the lower lip during a relaxed smile (Figs 22 and 23).

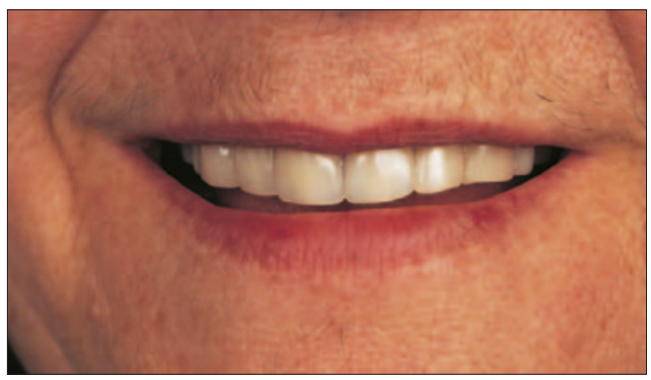

Fig. 22 Wear on acrylic teeth of removable full denture lacking parallelism with mandibular lip curvature

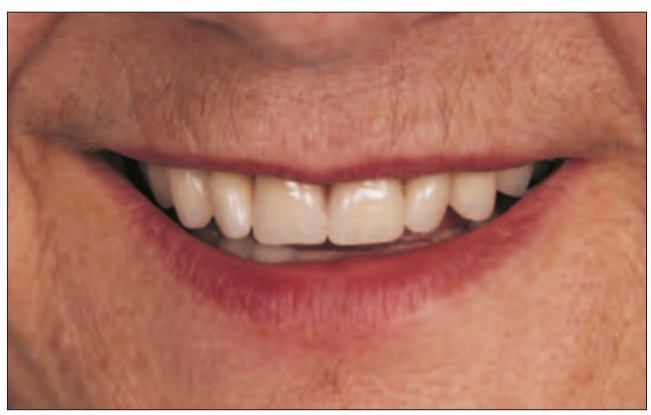

Fig. 23 Replacement of removable full denture (for patient in Fig. 22) with restitution of incisal plane, which is parallel with mandibular lip curvature
Similar to a painting, the anterior and lateral negative spaces act as a border to the dental elements, with the lips representing the picture frame. Anterior negative space is evident during both speech and laughter (Fig. 24), while bilateral negative spaces should be evident during a relaxed smile (Fig 25). These negative spaces provide cohesiveness to the dento-facial composition, and are also in the Golden proportion to the anterior dental segment. ${ }^{8}$

Fig. 24 Anterior negative space evident during speech and laughter
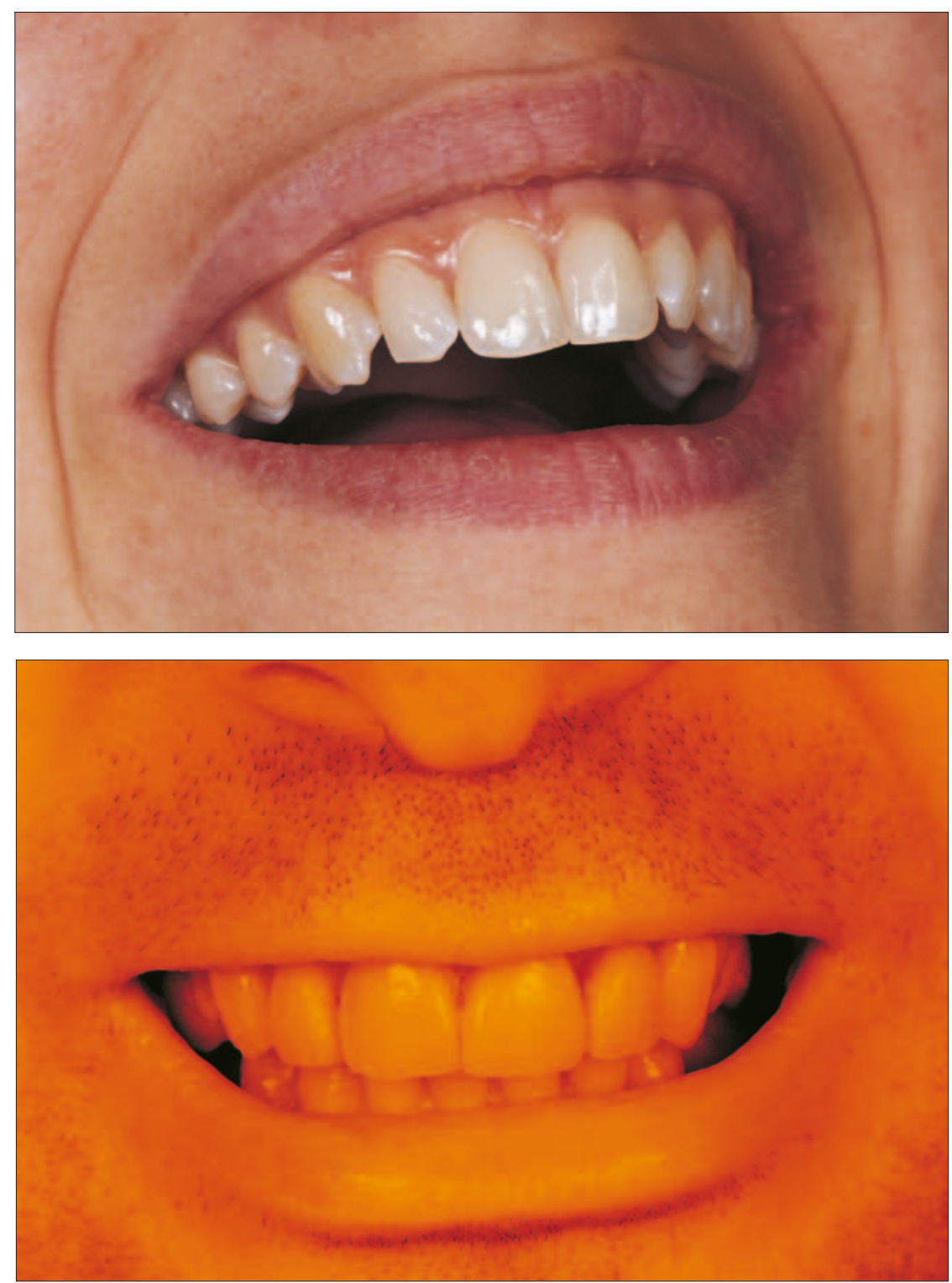

To summarise, there is no such thing as a perfect smile, however for the sake of defining guidelines for prosthodontics, the constituents of an ideal smile are when:

- The upper anterior teeth coincide with the curvature of the mandibular lip during a relaxed smile

- The corners of the lips are elevated to the same height on both sides (termed smile symmetry)

- Bilateral negative spaces are evident, separating the teeth from the corners of the lips.

Additionally, the contact points of the anterior dental segment should also coincide with the
Fig. 25 Infrared photograph showing bilateral negative spaces bounding the anterior dental segment 
incisal edges and with the curvature of the lower lip, thus enhancing the cohesiveness of the dentofacial composition (Figs 26 and 27).

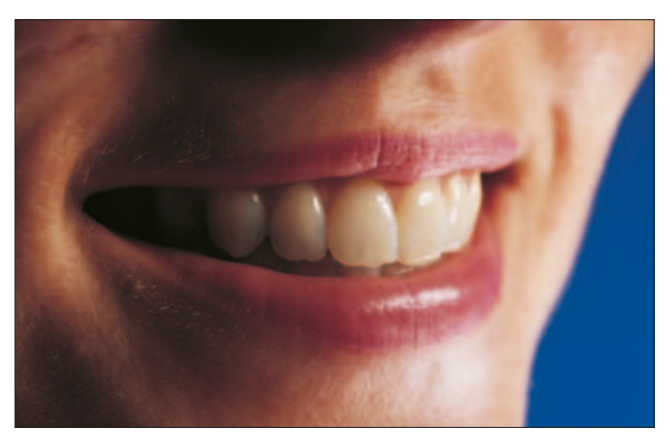

Fig. 26 Sagittal view of 'ideal' smile line (see text for explanation)

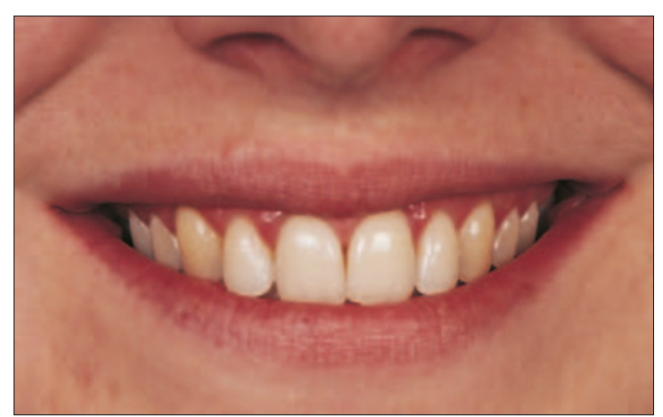

Fig. 27 Frontal view of 'ideal' smile line

(see text for explanation)

\section{CASE STUDY}

This case study highlights some of the points discussed above, and how these concepts can be used to achieve optimum dental aesthetics.

A 60-year-old lady attending the practice sought aesthetic improvement of her maxillary anterior segment. The preoperative views showed unsightly composite fillings in teeth 13, $12,11,21$ and 22. The left canine was the anterior abutment of a defective three-unit fixed partial denture (Figs 28 and 29).

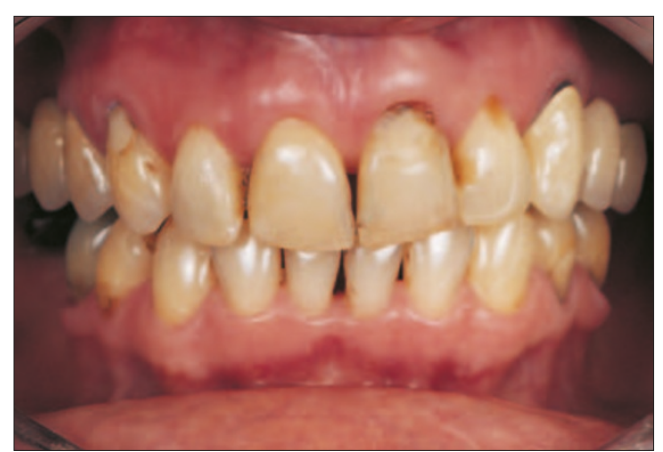

Fig. 28 Frontal view showing pre-operative status

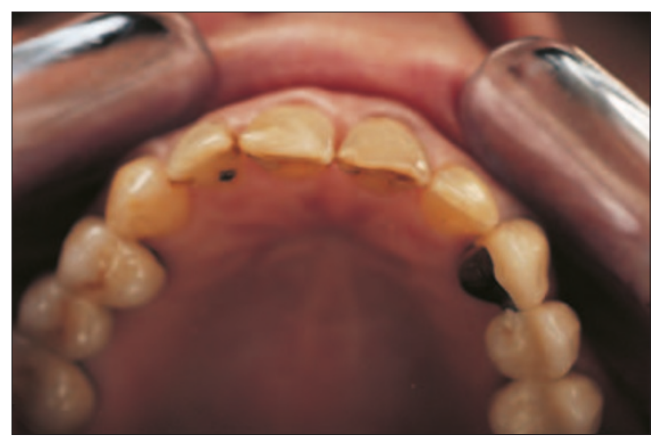

Additionally, the dentofacial composition revealed an undulating (roller-coaster) incisal plane, in conflict with the lower lip curvature, which was neither parallel to the commissural line (blue), nor the gingival exposure line (white) (Fig 30 and 31). Finally, in the dental composition, a myriad of flaws were evident, including diastemae, wear, and a lack of Golden proportion progression.

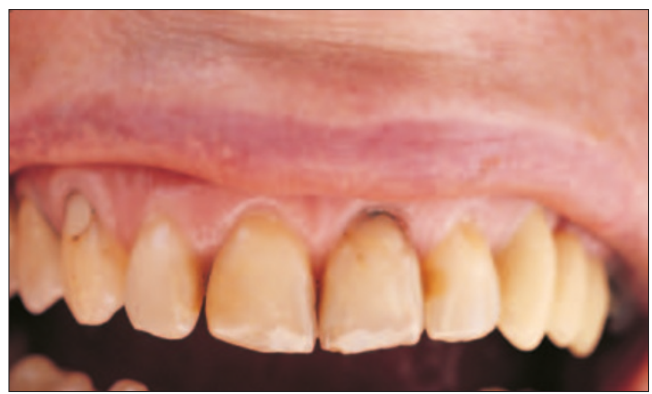

Fig. 30 Undulation, 'roller-coaster' incisal plane

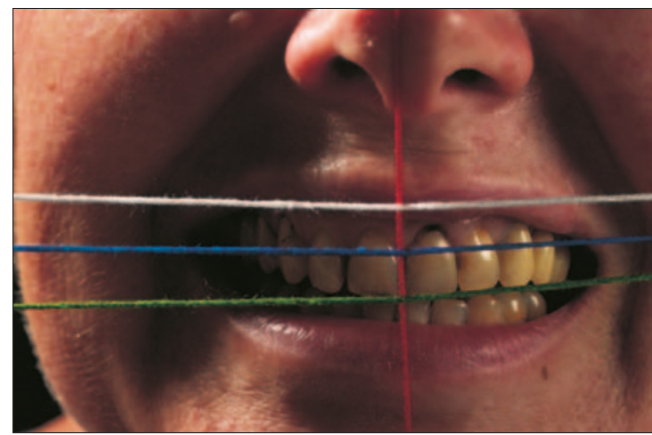

Fig. 31 Incisal plane (lime) is neither parallel to commissural (blue), nor gingival (white) lines

The treatment plan to resolve these aesthetic anomalies consisted of porcelain laminate veneers on teeth 13,12 and 22, full coverage ceramic crowns on the maxillary centrals, and a new three-unit ceramo-metal fixed partial denture with teeth 23 and 25 as abutments and 24 as the pontic. The completed tooth preparations for these restorations are shown in Figs 32-35.

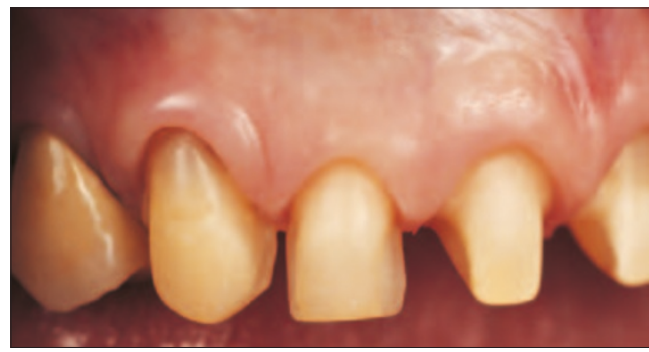

Fig. 32 Right lateral view of completed tooth preparation

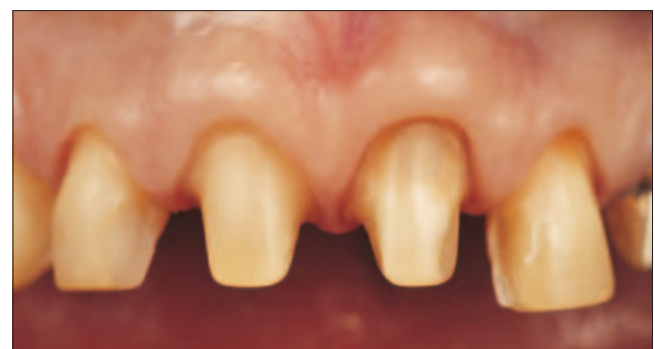

Fig. 33 Frontal view of completed tooth preparations 


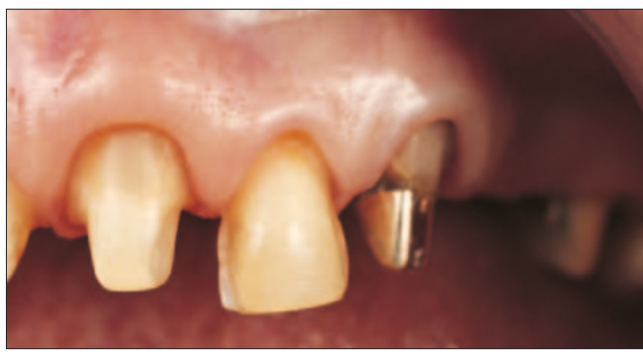

Fig. 34 Left lateral view of completed tooth preparations

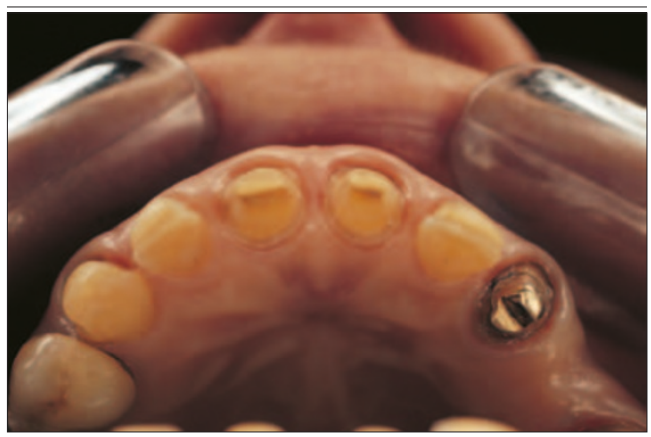

Fig. 35 Occlusal view of completed tooth preparations

After impressions and plaster casts were made (Figs 36 and 37), new restorations were fabricated to correct the anomalies mentioned above.

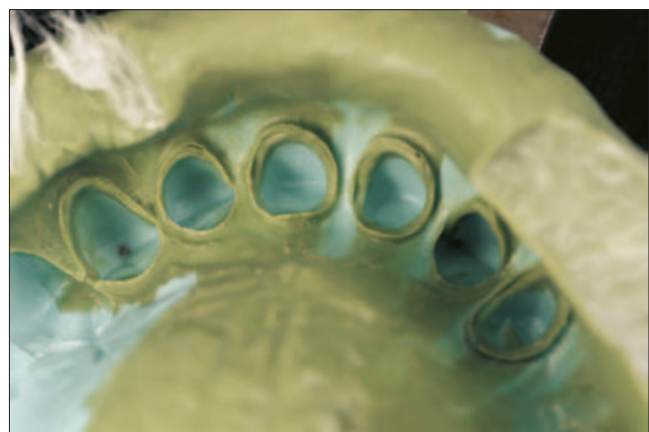

Fig. 36 Final impressions with an addition silicone material

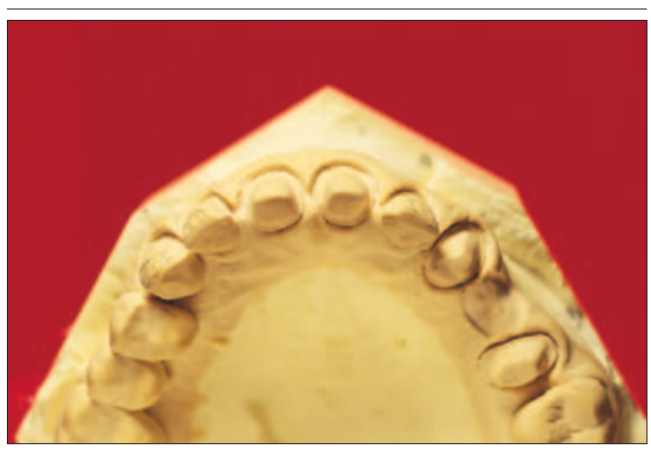

Fig. 37 Plaster cast of tooth preparations

The finished restorations show restitution of the maxillary incisal plane (Fig. 38). The ceramics in this case study were kindly done by Willi Geller (Oral Design, Zurich, Switzerland). Post cementation, the ceramic veneers and crowns blended impeccably with the fixed partial denture, with optimal gingival health depicted by formation of the gingival groove, knife edge gingival margins and stippling of the attached gingivae (Figs 39-41).
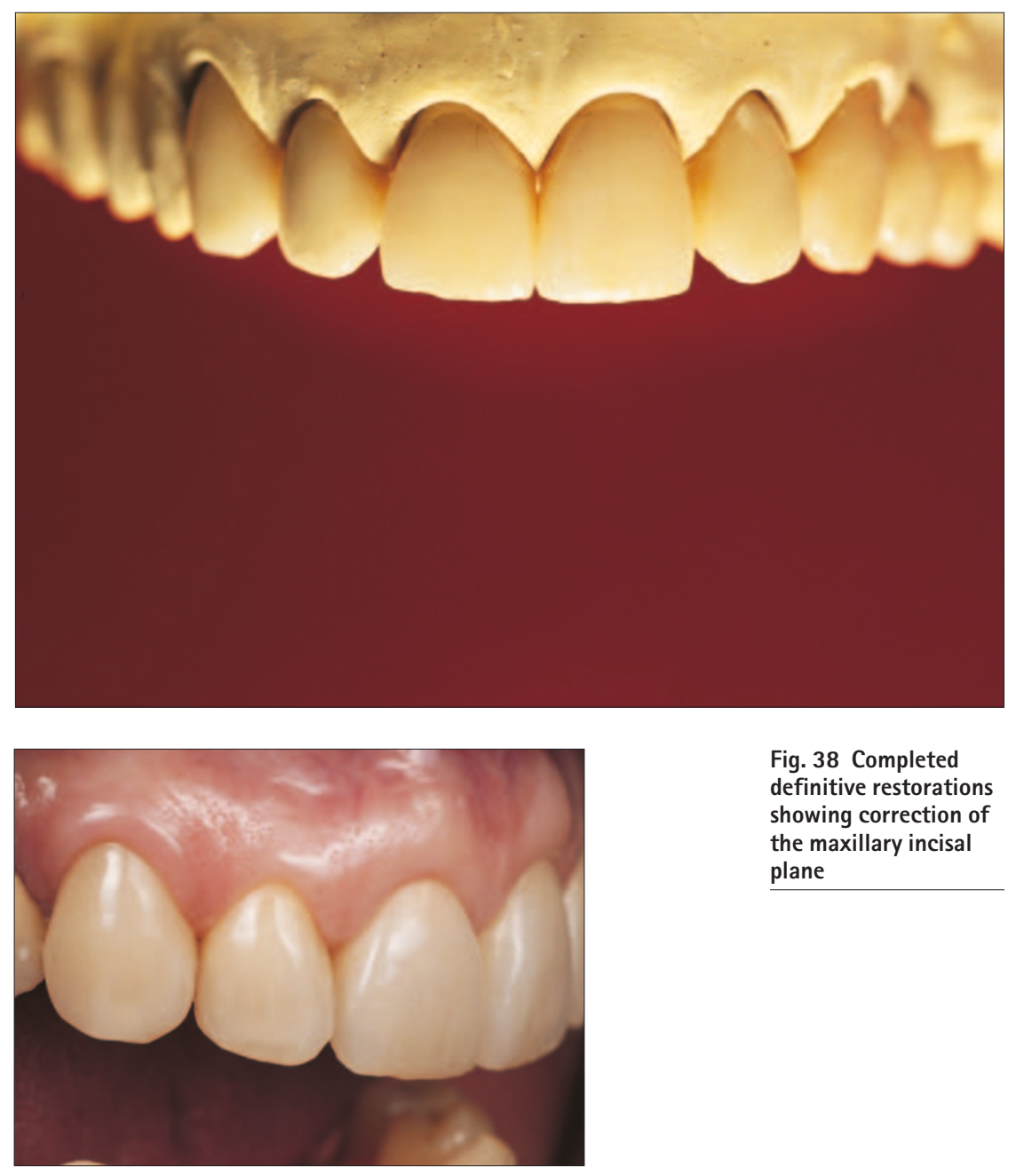

Fig. 38 Completed definitive restorations showing correction of the maxillary incisal plane

Fig. 39 Right lateral view of cemented prostheses

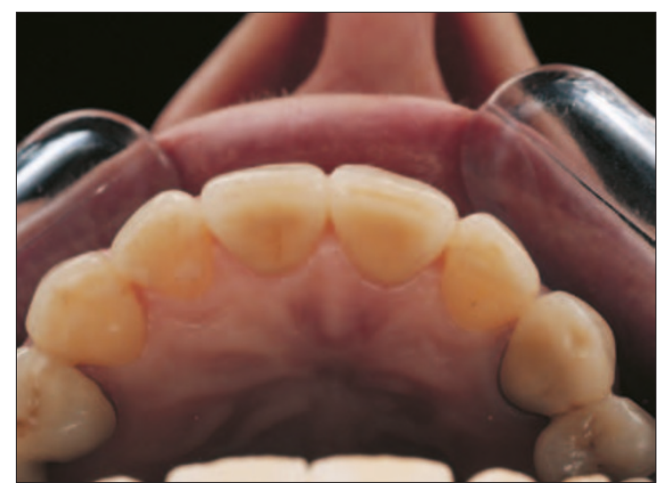

Fig. 40 Occlusal view of cemented prostheses

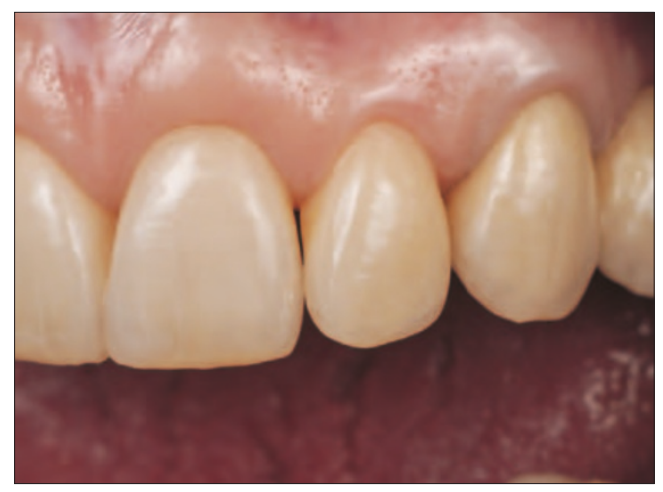

Fig. 41 Left lateral view of cemented prostheses 
The maxillary incisal plane was now parallel with the curvature of the mandibular lip and to the commisural (blue) and gingival exposure lines (Figs 42 and 43). Pre- and post-operative facial views showed the transformation and rejuvenation of the patient from being dejected and forlorn to a vivacious and confident persona (Figs 44 and 45).

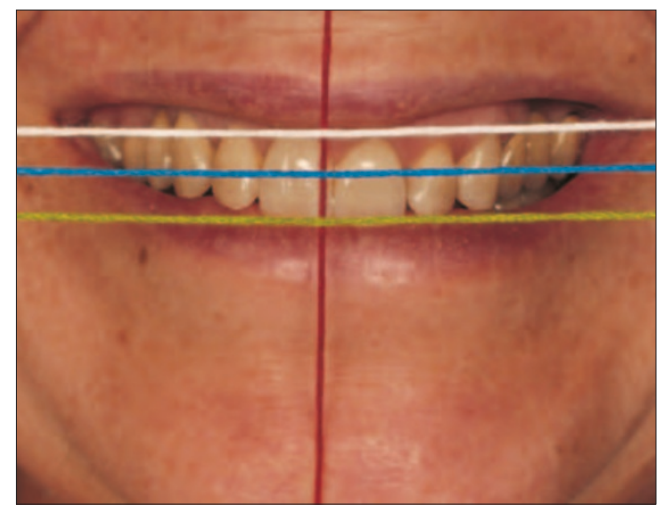

Fig. 42 Parallelism of incisal plane (lime) with commissural (blue) and gingival (white) lines (compare with Fig. 31)

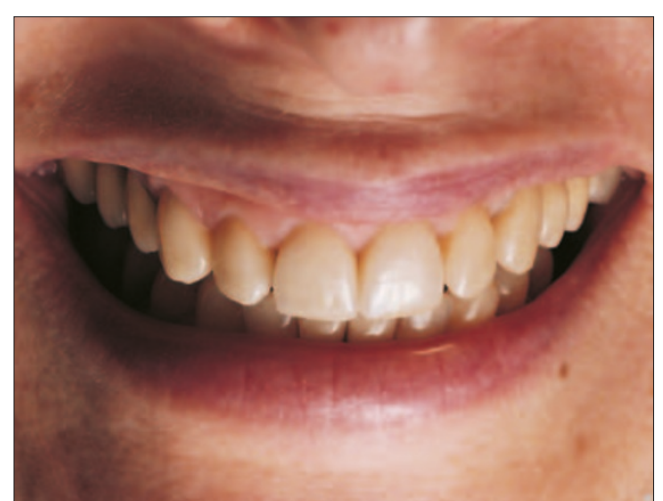

Fig. 43 Maxillary incisal plane coincident with curvature of mandibular lip (compare with Fig. 30)

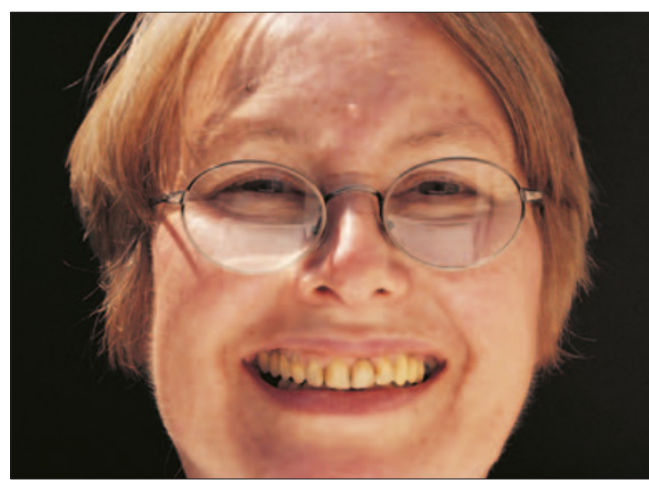

Fig. 44 Pre-operative facial view

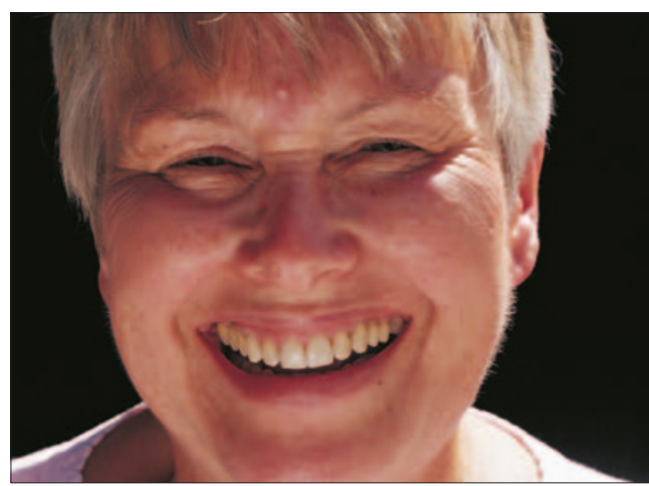

Fig. 45 Post-operative facial view

1. Vig R G, Brundo G C. The kinetics of anterior tooth display. J Prosthet Dent 1972; 39: 502

2. Ahmad I. Geometric considerations in anterior dental aesthetics: restorative principles. Practical periodontics and aesthetic dentistry, 1998; 10: 813-822.

3. Satre J P. The age of reason. Hamish Hamilton, 1947.

4. Rufenacht C R. Fundamental of aesthetics. Chicago: Quintessence Publishing Co Inc, II, 1990; 1: 18.

5. Heartwell C M. Syllabus of complete dentures. Philadelphia: Pa: Lea and Febiger, 1968

6. Dental Office Procedures. CA: Swissedent Foundation, 1990

7. Miller E C, Sodden E R, Jamison H C. A study of the relationship of the dental midline to the facial median line. J Prosthet Dent 1979; 41:657-660.

8. Levin El. Dental esthetics and the golden proportion. J Prosthet Dent 1978; 40: 244-252. 